\title{
Dihydroartemisinin inhibits tumor growth of human osteosarcoma cells by suppressing Wnt// -catenin signaling
}

\author{
YUELIANG LIU ${ }^{1 *}$, WENJUAN WANG ${ }^{1 *}$, JING XU $^{1}$, LI LI $^{1}$, QIAN DONG ${ }^{1}$, QIONG SHI ${ }^{1}$, GUOWEI ZUO ${ }^{1}$, \\ LAN ZHOU $^{1}$, YAGUANG WENG ${ }^{1}$, MIN TANG ${ }^{1}$, TONGCHUAN HE ${ }^{2}$ and JINYONG LUO ${ }^{1}$ \\ ${ }^{1}$ Key Laboratory of Diagnostic Medicine Designated by The Chinese Ministry of Education, Chongqing Medical University, \\ Chongqing 400016, P.R. China; ${ }^{2}$ Molecular Oncology Laboratory, Department of Surgery, \\ The University of Chicago Medical Center, Chicago, IL 60637, USA
}

Received June 17, 2013; Accepted July 12, 2013

DOI: $10.3892 /$ or.2013.2658

\begin{abstract}
Osteosarcoma (OS) is the most common type of bone cancer. Even with early diagnosis and aggressive treatment, the prognosis for OS is poor. In the present study, we investigated the proliferation and invasion inhibitory effect of dihydroartemisinin (DHA) on human OS cells and the possible molecular mechanisms involved. We demonstrated that DHA can inhibit proliferation, decrease migration, reduce invasion and induce apoptosis in human OS cells. Using an in vivo tumor animal model, we confirmed that DHA can prevent OS formation and maintain intact bone structure in athymic mice. In addition, we examined the possible molecular mechanisms mediating the function of DHA. We found that the total protein levels and transcriptional activity of $\beta$-catenin in OS cells are reduced by DHA treatment, and this may result from the increased catalytic activity of glycogen synthase kinase $3 \beta$ (GSK3 $\beta$ ). Moreover, the inhibitory effect of DHA on OS cells is reversed by overexpression of $\beta$-catenin, but is further enhanced by knockdown of $\beta$-catenin, respectively. Collectively, our results reveal that DHA can inhibit tumor growth of OS cells by inactivating Wnt/ $\beta$-catenin signaling. Therefore, DHA is a promising chemotherapy agent in the treatment of human OS.
\end{abstract}

\section{Introduction}

Osteosarcoma (OS) is one of the most common types of nonhematologic malignant bone cancer in humans, particularly in children and adolescents $(1,2)$. Although other types of cancer can eventually penetrate into parts of the skeleton, OS is one

Correspondence to: Dr Jinyong Luo, Key Laboratory of Diagnostic Medicine Designated by The Chinese Ministry of Education, Chongqing Medical University, 1 Yixueyuan Road, Chongqing 400016, P.R. China E-mail: luojinyong888@hotmail.com

*Contributed equally

Key words: dihydroartemisinin, osteosarcoma, Wnt/ß-catenin signaling, tumor growth of the few that actually initiate in bones and sometimes metastasize elsewhere, usually to the lungs (1-4). Approximately $80 \%$ of OS patients have metastatic disease at the time of diagnosis, and yet $10-15 \%$ of these lesions are detectable with current radiographic imaging modalities (4). The main clinical approach for OS patient is comprised of surgery with wide excision of the primary tumor and chemotherapy, a treatment which often proves difficult for patients due to the systemic toxicities of chemotherapy agents $(5,6)$. Chemotherapy drugs may cause hearing loss, anemia, abnormal bleeding, kidney and liver damage. Nevertheless, chemotherapy plays a major role in the treatment of OS currently (7-9). The effectiveness of chemotherapy in the prognosis of OS patients cannot be further improved simply by increasing the dose of chemotherapeutic drugs. Therefore, there is a great clinical need to explore new agents for the treatment of OS.

It is currently accepted that herbal and natural products are valuable resources for anticancer drugs $(10,11)$. Artemisinin (ART) is a natural product originally isolated from the plant Artemisia annua L., an herbal drug that has been used in traditional Chinese medicine for centuries $(12,13)$. Dihydroartemisinin (DHA) is a derivative of ART and is a potent anti-malarial drug with only mild adverse effects. Recent studies have shown that ART derivatives including DHA also have profound effects against human tumors including breast, pancreas, prostate and liver cancer with fewer toxic effects (14-18). However, the exact molecular mechanisms by which DHA exerts its anticancer effects remain to be fully investigated.

Here, we investigated the antitumor effect of DHA on human OS cells, as well as the possible molecular basis underlying the function. Our results strongly indicate that DHA can inhibit tumor growth of OS cells both in vitro and in vivo. The inhibitory effect of DHA on OS cells can be mediated by inactivating $\mathrm{Wnt} / \beta$-catenin signaling, which may be processed in part through increasing catalytic activity of glycogen synthase kinase $3 \beta$ (GSK3 $\beta)$.

\section{Materials and methods}

Cell culture and drug preparations. OS cell lines 143B, U2OS, SaoS2 and MG63 were obtained from the American 
Type Culture Collection, and maintained in Dulbecco's Modified Eagle's Medium (DMEM) with 10\% fetal bovine serum (FBS), $100 \mathrm{U} / \mathrm{ml}$ of penicillin and $100 \mu \mathrm{g} / \mathrm{ml}$ of streptomycin at $37^{\circ} \mathrm{C}$ in $5 \% \mathrm{CO}_{2}$. DHA was purchased from Sigma-Aldrich, dissolved in dimethyl sulfoxide (DMSO) for in vitro test, or prepared with $0.5 \%$ carboxymethyl cellulose sodium (CMC-Na) as suspension for in vivo experiments. Antibodies were from Santa Cruz Biotechnology, Inc., and Cell Signaling Technology. All other reagents were purchased form Sigma-Aldrich, unless otherwise indicated.

Crystal violet assay. OS cells were seeded in 24-well plates and treated with different concentrations of DHA or DMSO as control. At the scheduled time points, cells were stained with crystal violet to visualize the cell viability. For quantification, crystal violet in the stained cells was extracted with $1 \mathrm{ml}$ $20 \%$ acetic acid at room temperature for $20 \mathrm{~min}$ with shaking.

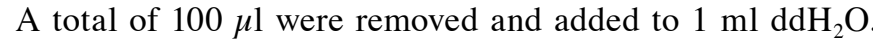
Absorbance at $570 \mathrm{~nm}$ was measured (19). Each assay was performed in triplicate.

MTT proliferation assay. 143B cells were seeded in 96-well plates and treated with different concentrations of DHA. Cell proliferation was assessed by MTT assay at the scheduled time points. Optical absorbance was measured at $570 \mathrm{~nm}$ using a 96-well microplate reader. All experiments were performed in triplicate.

Colony formation assays. One day prior to DHA treatments, 200 cells were seeded into 6-well plates in complete DMEM. The cells were then treated with variable concentrations of DHA and cultured in complete DMEM for up to 14 days. Colonies were visualized by crystal violet staining and counted. The colony forming rate was obtained by the following calculation: (colony number/seeded cell number) x100\%. All experiments were performed in triplicate.

Transwell migration and invasion assay. The migration and invasion assay was performed as previously described $(20,21)$. For the migration assay, $143 \mathrm{~B}$ cells were seeded at $2 \times 10^{5} /$ well in the upper chamber of type I-collagen-coated 24-well culture inserts. For the invasion assay, the upper side of the insert was coated with extracellular matrix (ECM) gel. DHA was added to the lower chamber. After $24 \mathrm{~h}$, the cells were dried for $5 \mathrm{~min}$, fixed with dehydrated alcohol, and stained with hematoxylin-eosin. The transmembrane cells were counted. All experiments were performed in triplicate.

Hoechst apoptosis staining. 143B cells were plated in 24-well plates and treated with different concentrations of DHA or DMSO. After maintaining at $37^{\circ} \mathrm{C}$ in $5 \% \mathrm{CO}_{2}$ for $48 \mathrm{~h}$, cells were collected and subjected to Hoechst 33258 staining. The apoptotic cells were visualized under fluorescence microscopy as previously reported (22). Each assay was carried out in triplicate.

Western blot assay. 143B cells were plated in 24-well plates and treated with different concentrations of DHA or DMSO. The cells were then collected and lysed in Laemmli buffer. Cleared total cell lysate was denatured by boiling and loaded onto a
4-20\% gradient SDS-PAGE. Following electrophoretic separation, proteins were transferred to an Immobilon-P membrane. The membrane was blocked with SuperBlock blocking buffer and probed with the primary antibody, followed by incubation with a secondary antibody-conjugated with horseradish peroxidase. The proteins of interest were detected by using SuperSignal West Pico Chemiluminescent Substrate kit. Each assay was carried out in triplicate.

Luciferase reporter assay. 143B cells were seeded in T-25 flask and transfected with $3 \mu \mathrm{g}$ of Top-luc luciferase reporter plasmids. Twelve hours later, trypsinized cells were seeded to 24-well plates and were then treated with DHA or DMSO. At the scheduled time points, cells were lysed and subjected to luciferase assays using luciferase assay kit. Luciferase activity was normalized by total cellular protein concentrations of the samples. Each assay was performed in triplicate.

Establishment of orthotopic OS tumor animal model. All animal experiments were approved by the Institutional Animal Care and Use Committee (IACUC) of Chongqing Medical University. Athymic mice (female, 4-6 weeks old, 5/group) were ordered from the Animal Centre of Chongqing Medical University (Chongqing, China). 143B cells were collected and resuspended in cold $\left(4^{\circ} \mathrm{C}\right)$ phosphate buffer solution (PBS) to a final density of $2 \times 10^{7}$ cells $/ \mathrm{ml}$. Cells in $50 \mu 1$ of cold PBS were injected into the proximal tibia of athymic mice. Animals were then treated with either different doses of DHA $(5,10$ and $20 \mathrm{mg} / \mathrm{kg}$ ) or solvent by intragastric administration, once a day until the 37th day. The tumor size was measured every day from the 1st week. Animals were sacrificed at 37 days after injection. The tumor samples were retrieved for histological evaluation.

Statistical analysis. Data presented are the results of at least 2 independent experiments performed in triplicate. Microsoft Excel was employed to calculate the standard deviations. The differences were analyzed using the Student's t-test. $\mathrm{p}<0.05$ was considered to indicate a statistically significant difference.

\section{Results}

DHA inhibits the proliferation of OS cells. We first employed crystal violet staining to test the proliferation inhibitory effect of DHA on human OS cells. The results showed that DHA inhibited the proliferation of 143B OS cells effectively in a concentration-dependent manner, even at the minimum concentration of $2.5 \mu \mathrm{M}$ (Fig. 1A and B). Similar results were obtained in other commonly-used OS cell lines, including U2OS, SaoS2 and MG63 (Fig. 1C and D). Moreover, the inhibitory effect of DHA on 143B cell proliferation was further confirmed by MTT assay (Fig. 1E) and colony formation assay (Fig. 1F and G). Proliferating cell nuclear antigen (PCNA) was a simple but potent marker for the assessment of OS growth (23). Therefore, we also tested the level of PCNA with western blotting. The results showed that the level of PCNA in 143B cells was decreased by DHA as well (Fig. 1H). Collectively, these results suggest that DHA effectively inhibits the proliferation of OS cells. 

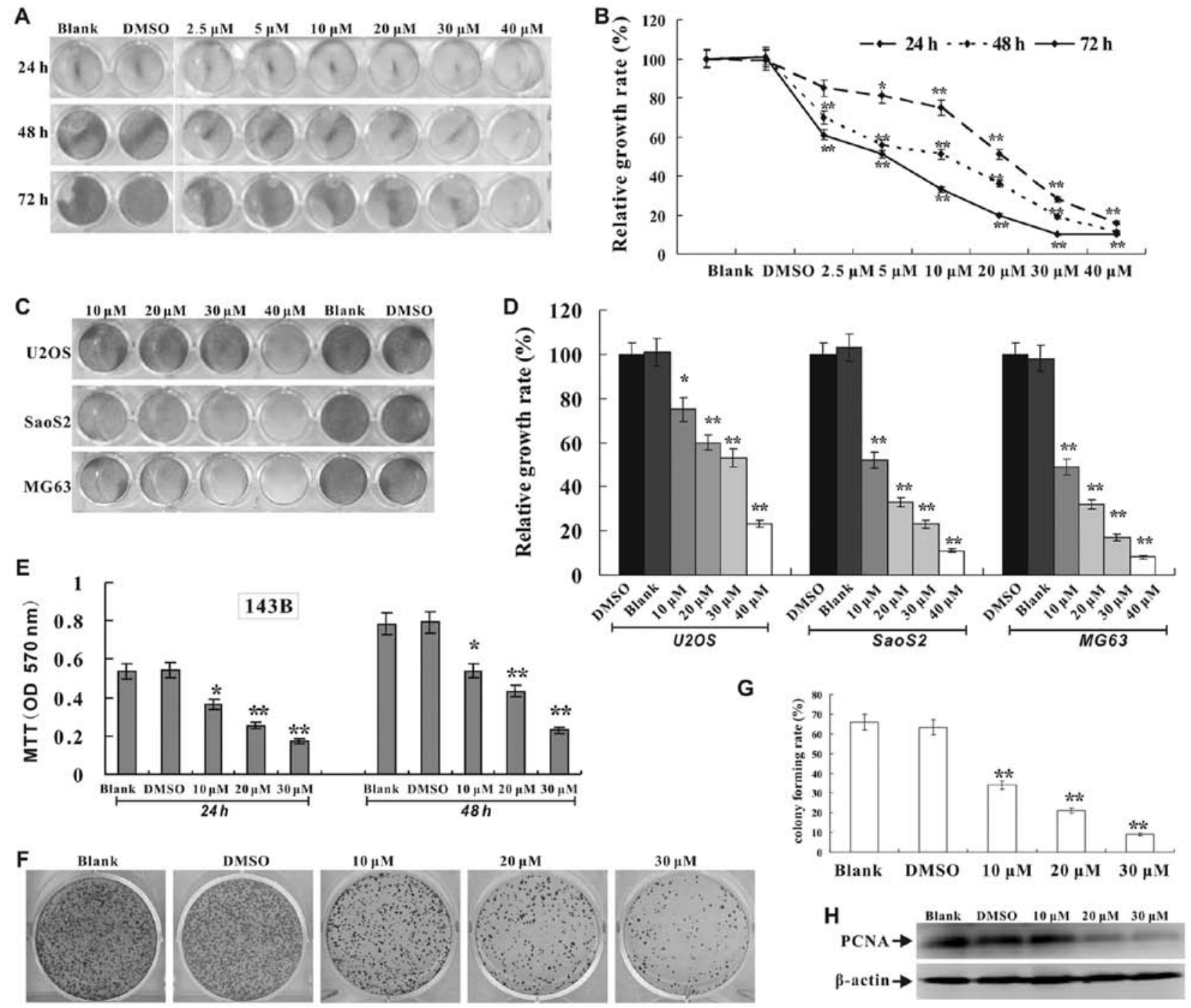

Figure 1. DHA inhibits the proliferation of human OS cells. (A) The crystal violet staining results in 143B OS cells. 143B cells were seeded in 24-well plates and treated with DHA at the indicated concentrations. At 24, 48 and $72 \mathrm{~h}$ after treatment, the cells were stained with crystal violet. (B) Quantitative results of crystal violet staining in 143B cells. The assay was performed in triplicate. " $\mathrm{p}<0.05$ vs. blank and DMSO; ${ }^{* *}<0.01$ vs. blank and DMSO. (C) The crystal violet staining results in U2OS, SaoS2 and MG63 OS cells. U2OS, SaoS2 and MG63 OS cells were seeded in 24-well plates and treated with DHA at the indicated concentrations. At $72 \mathrm{~h}$ after treatment, the cells were stained with crystal violet. (D) Quantitative results of crystal violet staining in U2OS, SaoS2 and MG63 OS cells. The assay was performed in triplicate. " $\mathrm{p}<0.05$ vs. blank and DMSO; ** $\mathrm{p}<0.01$ vs. blank and DMSO. (E) MTT results in $143 \mathrm{~B}$ cells. 143B cells were seeded in 96-well plates and treated with DHA as the indicated concentrations. At 24 and $48 \mathrm{~h}$ after treatment, the cells were subjected to MTT assay. The assay was performed in triplicate. " $\mathrm{p}<0.05$ vs. blank and DMSO; ${ }^{* *} \mathrm{p}<0.01$ vs. blank and DMSO. (F) The colony formation results (crystal violet staining) in 143B cells treated with DHA at the indicated concentrations. At 14 days after treatment, the formed colonies were subjected to crystal violet staining. (G) Quantitative results of colony formation rates (\%) in 143B cells. The assay was performed in triplicate. "p $<0.05$ vs. blank and DMSO; ** $\mathrm{p}<0.01$ vs. blank and DMSO. (H) Western blot assay for the PCNA protein level in 143B cells. Cells were seeded in 6-well plates and treated with the indicated concentrations of DHA or DMSO for $24 \mathrm{~h}$ and were then harvested for western blot assay. DHA, dihydroartemisinin; OS, osteosarcoma; DMSO, dimethyl sulfoxide; PCNA, proliferating cell nuclear antigen.

DHA inhibits the migration and invasion of OS cells. Cell migration and invasion play a crucial role in the process of tumor metastasis. Herein, transwell assay without/with ECM was used to assess the alteration in cellular migration and invasion induced by DHA in OS cells. As shown in Fig. 2A and B, DHA inhibited the number of migrated OS cells in a dose-dependent manner. We also found that when 143B cells were treated with DHA, the number of invaded cells across the ECM-coated membranes decreased accordingly (Fig. 2C and D). Moreover, as illustrated in Fig. 2E, DHA reduced the protein level of matrix metalloproteinase 9 (MMP9), vascular endothelial growth factor (VEGF) and cyclooxygenases 2 (COX2), which have been proved to highly associate with migration and inva- sion in cancer metastasis (24-26). These results demonstrate that DHA effectively attenuates the migrating and invasiveness phenotype of OS cell in vitro. This function may at least partly result from the reduction of MMP9, VEGF and COX2 proteins in OS cells.

DHA induces apoptosis in OS cells. We next investigated whether DHA induces OS cells to undergo apoptosis with Hoechst 33258 staining assay. The result showed that the apoptotic cells in the DHA-treated group were more pronounced than those of the blank or DMSO group (Fig. 3). Furthermore, we found that the level of cleaved caspase 3 , a well-established central executor in cell apoptosis, was increased by DHA in 


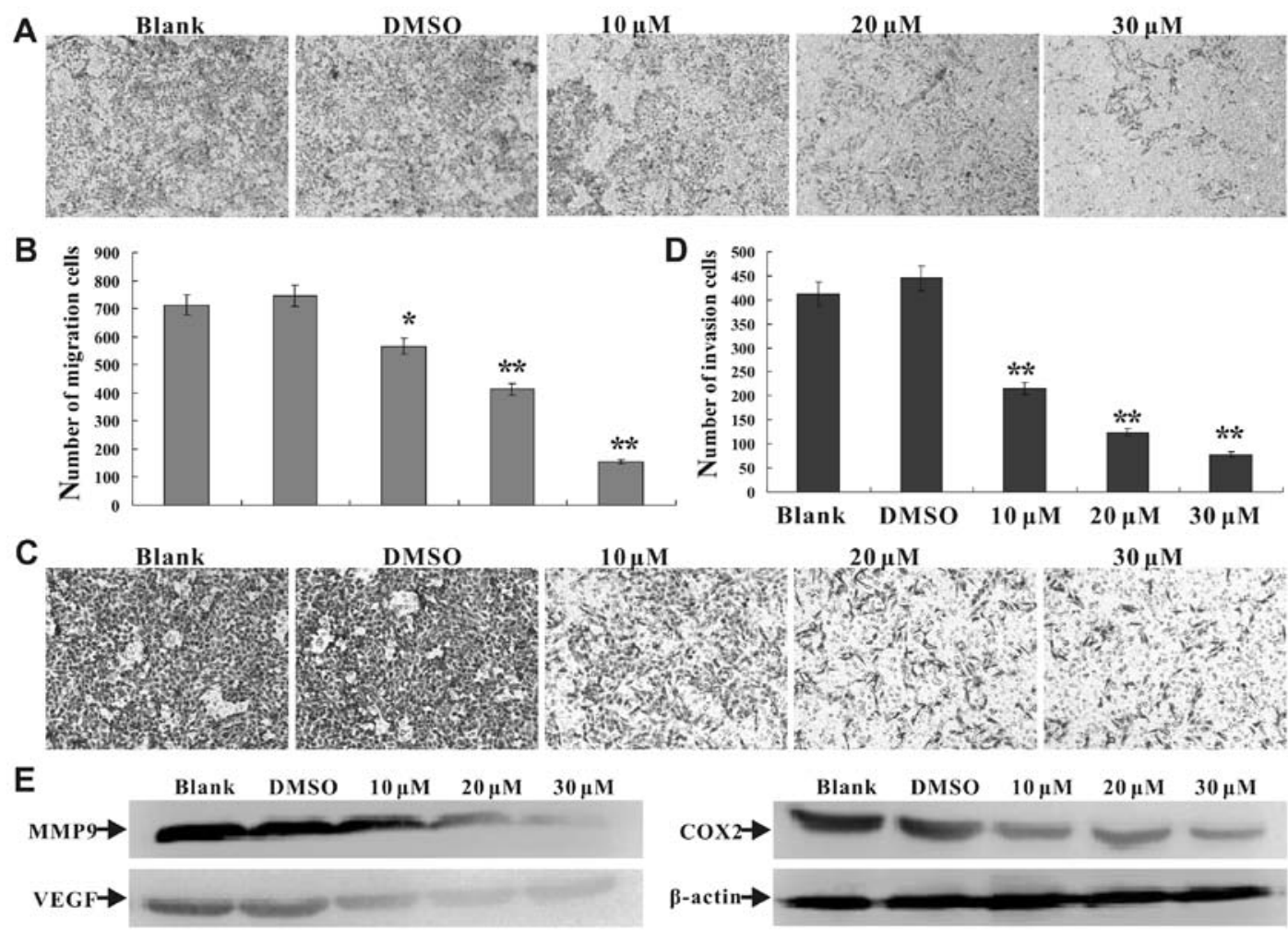

Figure 2. DHA inhibits migration and invasion of OS cells. (A) The transwell migration assay (without EMC) in 143B cells treated with DHA at the indicated concentrations. Migrated cells were fixed, stained and microphotographed. Representative results are shown. Magnification, x100. (B) The quantitative results of transwell migration assay in 143B cells. Migrated cells were counted as the average number of migrated cells per 5-0 high power fields. The assay was performed in triplicate. " $\mathrm{p}<0.05$ vs. blank and DMSO; ${ }^{* *} \mathrm{p}<0.01$ vs. blank and DMSO. (C) The transwell invasion assay (with EMC) in 143B cells treated with DHA at the indicated concentrations. Invaded cells were fixed, stained and microphotographed. Representative results are shown. Magnification, $\mathrm{x} 100$. (D) The quantitative results of transwell invasion in 143B cells. Invaded cells were counted as the average number of migrated cells per 5-10 high power fields. The assay was performed in triplicate. ${ }^{*} \mathrm{p}<0.05$ vs. blank and DMSO; ${ }^{* *} \mathrm{p}<0.01$ vs. blank and DMSO. (E) Western blot assay for the MMP9, VEGF and COX2 proteins in 143B cells. Cells were seeded in 6-well plates and treated with the indicated concentrations of DHA or DMSO for $24 \mathrm{~h}$, then harvested for western blot assay. DHA, dihydroartemisinin; OS, osteosarcoma; DMSO, dimethyl sulfoxide; MMP9 metalloproteinase 9; VEGF, vascular endothelial growth factor; COX2, cyclooxygenases 2 .

a concentration-dependent manner. DHA also disturbed the balance between proapoptotic protein Bad and anti-apoptotic protein $\mathrm{Bcl} 2$, led to an increase in Bad but a reduction in $\mathrm{Bcl} 2$ protein (Fig. 2C). The data indicate that DHA is capable of inducing and promoting apoptosis, a characteristic shared by most of the currently used chemotherapy agents $(27,28)$.

DHA inhibits tumor growth of OS cells in vivo. The above data demonstrate that DHA inhibited tumor growth of OS cells in vitro. We sought to confirm these findings in an orthotopic OS tumor animal model in vivo. 143B cells were implanted into the proximal tibia of athymic mice under DHA treatment, and tumor size was monitored. As shown in Fig. 4A, the DHA treatment group exhibited significantly decreased tumor size, compared with the control group. At 37 days, the animals were euthanized and the tumor masses were retrieved (Fig. 4B). The H\&E staining results showed that sample in the control group was more aggressive than that of the DHA-treated groups, the OS cells invaded and replaced almost all the bone at primary site (Fig. 4C). However, the samples retrieved from the DHA group displayed relative intact bone structure and less tumor cells, particularly in the high dose group (Fig. 4C). These results confirm that DHA inhibits OS tumor growth in vivo.
DHA suppresses Wnt/ $\beta$-catenin signaling in OS cells. We found that DHA inhibits tumor growth of OS cells both in vitro and in vivo. Next, we sought to examine the possible mechanism behind the anticancer activity of DHA in OS cells. Western blot results showed that p-P53, P53 and MDM2 were not altered under DHA treatment, indicating that the level of these molecules is possibly not related to the anticancer effects of DHA in OS cells (Fig. 5A). However, total $\beta$-catenin protein was effectively reduced by DHA, as were the $\beta$-catenin targets C-myc and cyclin D1 (Fig. 5A). Furthermore, we found that DHA was able to inhibit reporter activity of TOP-Luc, which contains TCF/LEF-responsive elements and reflects $\beta$-catenin transcriptional activity (Fig. 5B). These results indicate that DHA suppresses Wnt $/ \beta$-catenin signaling in OS cells. To investigate how DHA reduces the $\beta$-catenin protein in OS cells, we employed western blot assay to detect the activation status of GSK $3 \beta$ in OS cells. We found that DHA increased GSK $3 \beta$ activity by suppressing phosphorylation of GSK $3 \beta$ at serine 9 (Ser 9), which has been proved to be an inactive form of GSK3 $\beta$ (Fig. 5A). Notably, we observed that the level of DVL proteins, a main switchboard of Wnt/ $\beta$-catenin signaling, is decreased by treatment of DHA as well (Fig. 5A). These findings suggest that downregulation of $\beta$-catenin protein in OS by DHA resulted from upregulation of GSK $3 \beta$ activity and destabilize the $\beta$-catenin. 


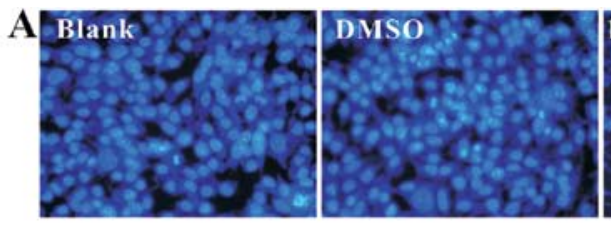

B

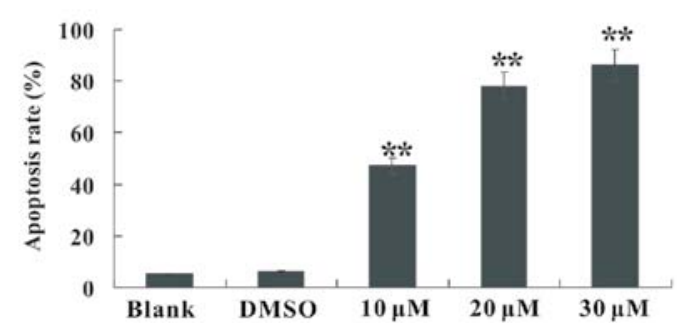

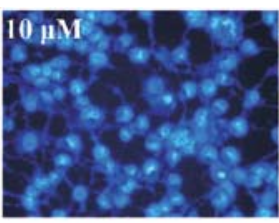
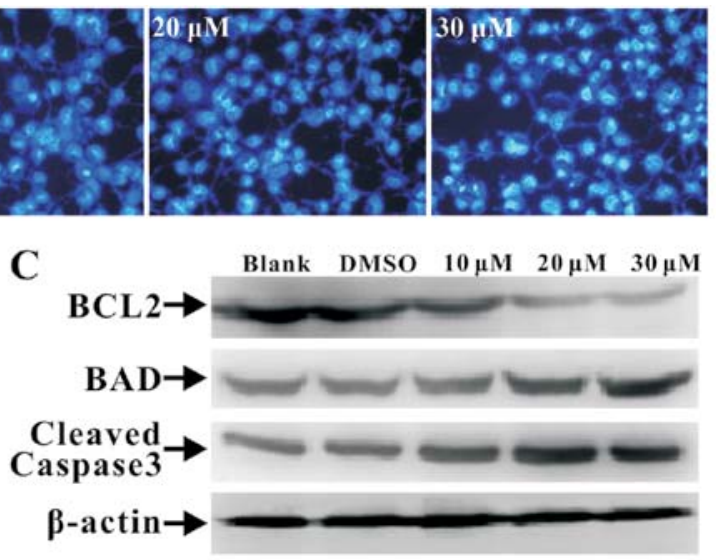

Figure 3. DHA induces apoptosis in human OS cells. (A) The Hoechst 33258 staining results in 143B cells. Cells were seeded in 24-well plates and treated with the indicated concentrations of DHA for $24 \mathrm{~h}$, then harvested and stained with Hoechst 33258. Representative results are shown. Magnification, x100. (B) The quantitative results of Hoechst 33258 staining results. The assay was performed in triplicate. ${ }^{*} \mathrm{p}<0.05$ vs. blank and DMSO; ${ }^{* * *}$ p $<0.01$ vs. blank and DMSO. (C) Western blot assay for Bad, Bcl2 and cleaved caspase 3 proteins in 143B cells. Cells were seeded in 6-well plates and treated with the indicated concentrations of DHA for $24 \mathrm{~h}$, then harvested for western blot assay. DHA, dihydroartemisinin; OS, osteosarcoma; DMSO, dimethyl sulfoxide.
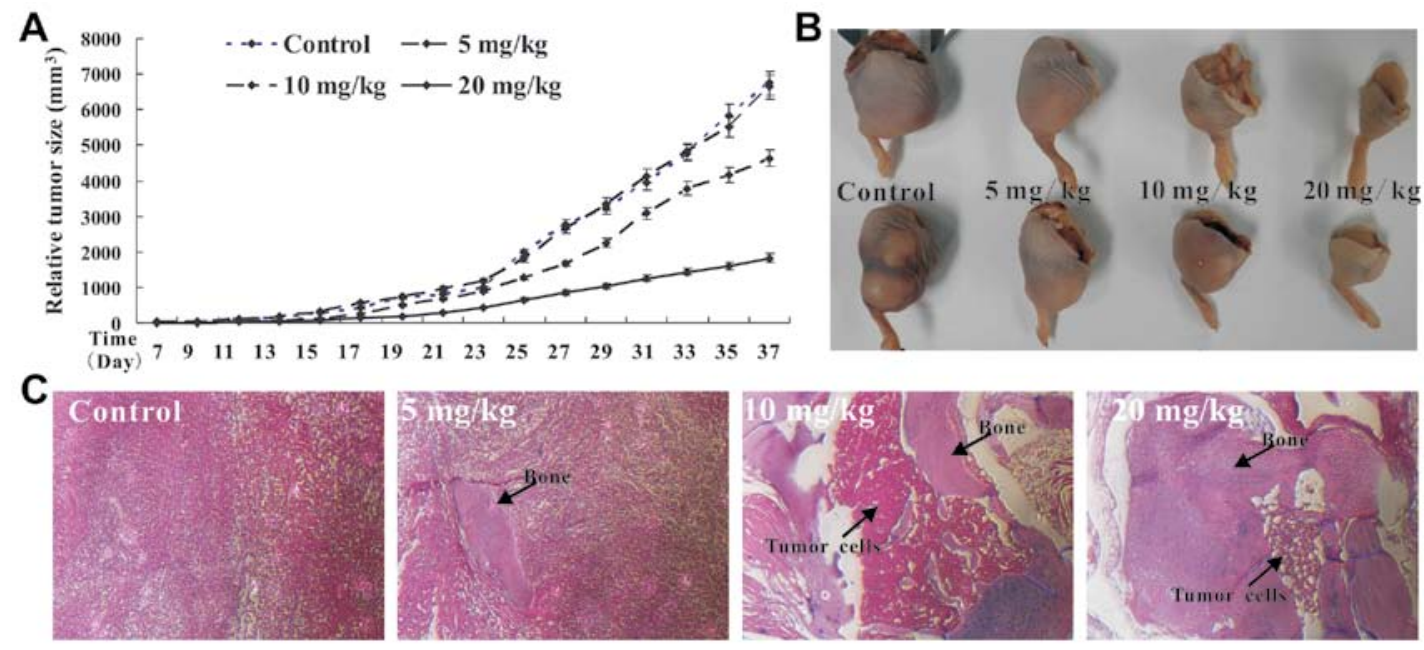

Figure 4. DHA inhibits OS tumor growth in vivo. (A) The change of tumor size with time. (B) Retrieved tumor samples from orthotopic OS tumor animal model. (C) H\&E staining results of the retrieved tumor sample. Representative results are shown. Magnification, x200. DHA, dihydroartemisinin; OS, osteosarcoma; H\&E; hematoxylin and eosin.

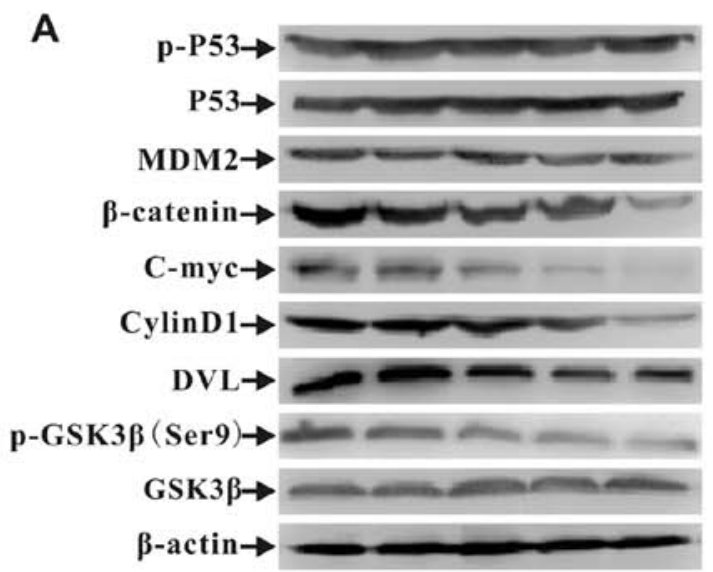

B

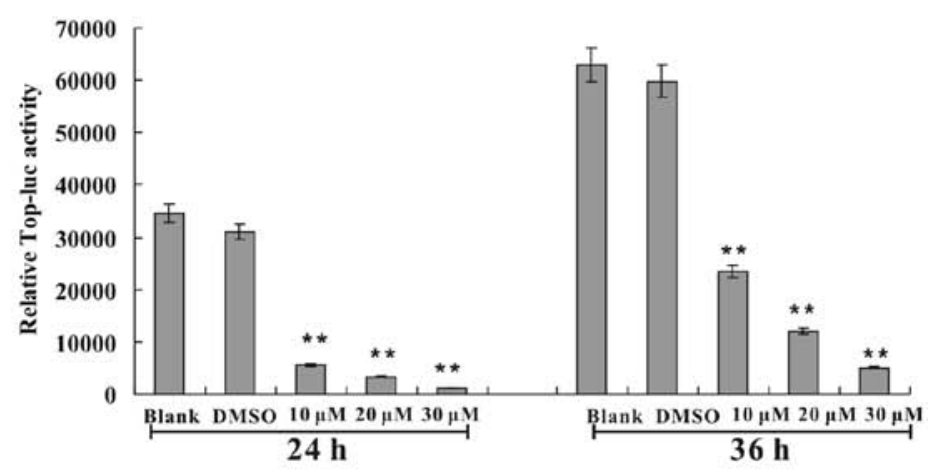

Figure 5. DHA suppresses Wnt/ $\beta$-catenin signaling in human OS cells. (A) Western blot assay for p-P53, P53, MDM2, $\beta$-catenin, c-myc, cyclin D1, DVL, p-GSK3 $\beta$ (Ser 9) and GSK3 $\beta$ in 143B cells. Cells were seeded in 6-well plates and treated with the indicated concentrations of DHA for $24 \mathrm{~h}$ and were then harvested for western blot assay. (B) $\beta$-catenin-controlled TOP-Luc reporter results in 143B cells. Cells were seeded in 6-well plates and were treated with the indicated concentrations of DHA for 24 or $36 \mathrm{~h}$, then harvested for luciferase activity test. The assay was performed in triplicate. ${ }^{* *} \mathrm{p}<0.01 \mathrm{vs}$. blank and DMSO. DHA, dihydroartemisinin; OS, osteosarcoma; GSK3 $\beta$, glycogen synthase kinase $3 \beta$; Ser 9, serine 9; DMSO, dimethyl sulfoxide. 
A

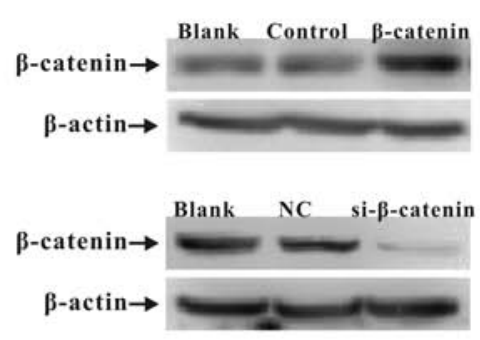

C

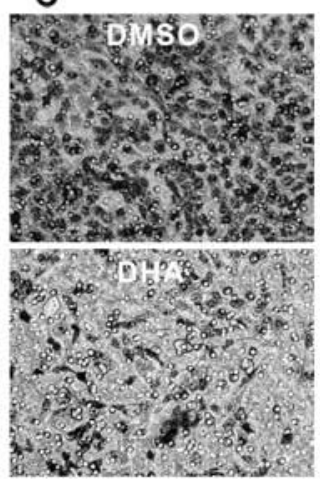

B
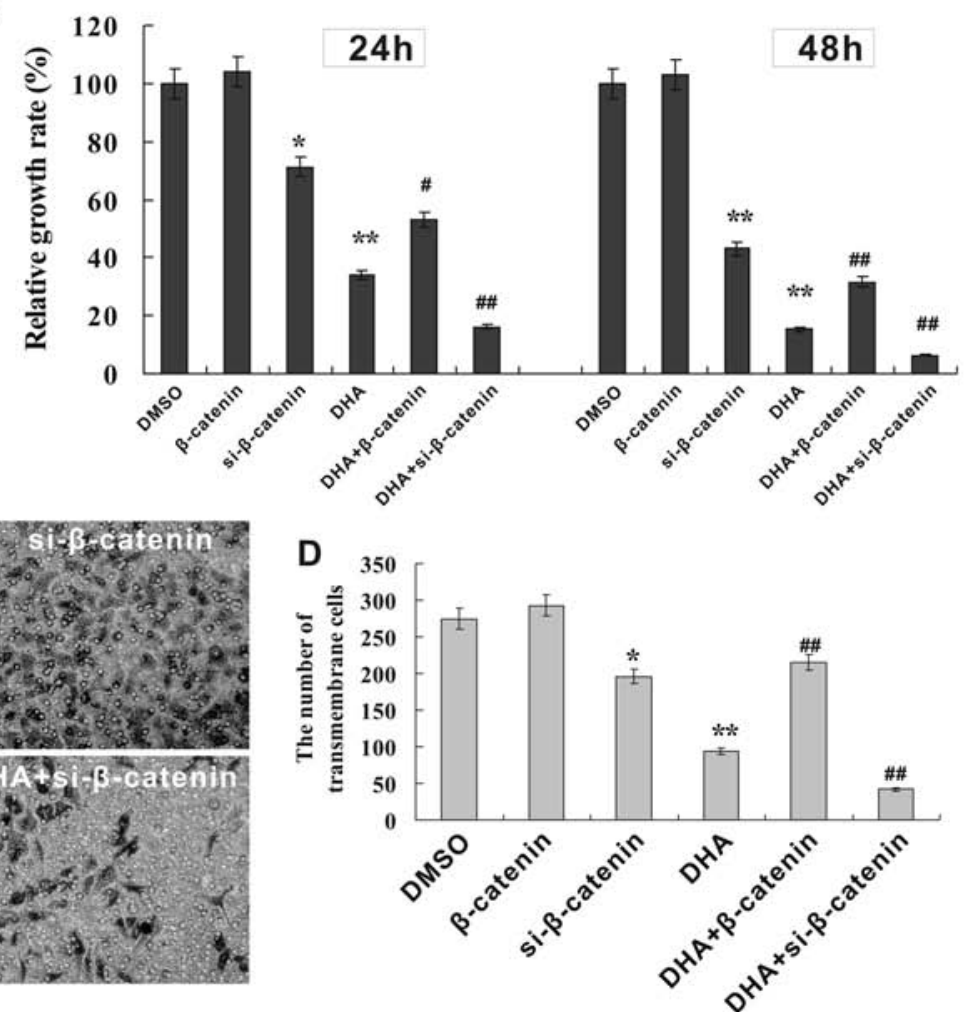

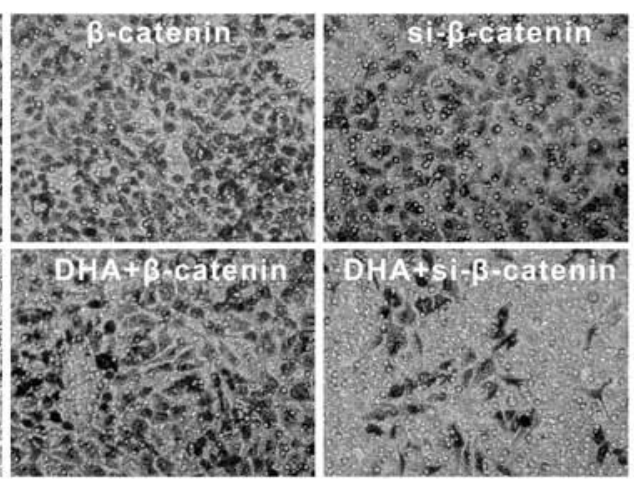

Figure 6. The function of $\beta$-catenin in the inhibitory effects of DHA on OS cells. (A) Western blot assay for exogenous expression and knockdown of $\beta$-catenin in 143B cells. (B) The quantitative results of 143B growth rate (crystal violet staining). Cells were seeded in $24-w e l l$ plates and treated with DHA and with/ without exogenous expressed $\beta$-catenin or knockdown of $\beta$-catenin. At $24 \mathrm{~h}$ after treatment, the cells were stained with crystal violet and growth rate was quantified. The assay was performed in triplicate. ${ }^{*} \mathrm{p}<0.05$ vs. DMSO; ${ }^{* *} \mathrm{p}<0.01$ vs. DMSO. ${ }^{\#} \mathrm{p}<0.05$ vs. DHA; ${ }^{\# \#}$ p $<0.01$ vs. DHA. (C) The transwell invasion results (with EMC) in 143B cells. Cells were seeded in 24-well plates and treated with DHA and with/without exogenous expressed $\beta$-catenin or knockdown of $\beta$-catenin. Invaded cells were fixed, stained and microphotographed. Representative results are shown. Magnification, x100. (D) The quantitative transwell invasion results in 143B cells. Invaded cells were counted as the average number of migrated cells per 5-10 high power fields. The assay was performed in triplicate. ${ }^{*}$ p $<0.05$ vs. DMSO; ${ }^{* *}$ p $<0.01$ vs. DMSO; ${ }^{\# \#}$ p $<0.01$ vs. DHA. DHA, dihydroartemisinin; OS, osteosarcoma; DMSO, dimethyl sulfoxide.

To validate the role of $\beta$-catenin in the inhibitory effect of DHA on OS cells, the overexpression of exogenous $\beta$-catenin or knockdown of $\beta$-catenin in OS cells was achieved respectively (Fig. 6A). The proliferation of 143B cells was suppressed by knockdown of $\beta$-catenin, although it was not altered by exogenous $\beta$-catenin (Fig. 6B). Markedly, we found that exogenous expression of $\beta$-catenin attenuated the growth inhibitory function of DHA, whereas knockdown of $\beta$-catenin enhanced this function in 143B cells, suggesting that decrease of $\beta$-catenin protein plays an important role in the anti-proliferation function of DHA in OS cells (Fig. 6B). In the transwell invasion assay, we observed that the invasion suppressive effect of DHA was neutralized by exogenous expression, but augmented by $\beta$-catenin knockdown (Fig. 6C and D). Collectively, the above data indicate that the inhibitory effect of DHA on OS cells may result from the inactivation of $\mathrm{Wnt} / \beta$-catenin signaling.

\section{Discussion}

OS is a common primary bone cancer with considerable morbidity and mortality. The current clinical strategies for OS include surgical resection, radiotherapy and chemotherapy. However, OS patients still have a poor prognosis under these aggressive treatments (29). Thus, there is an urgent need to develop more effective therapies for OS treatment. Herbal and natural products have been proved to be highly suitable sources for anticancer drugs (10,11,14-18). DHA, a derivative of ART, was initially used as an anti-malarial. However, recent studies indicated that DHA is capable of improving immunity, preventing inflammation, regulating cell differentiation and inhibiting cancer growth with low host toxicity (30-34). The anticancer effect of DHA is partly associated with iron homeostasis (33). Moreover, DHA is shown to activate p38 and ERK1/2, indicating these signal molecules are relevant to the anticancer effects of DHA (34). Despite these findings, the molecular mechanism through which DHA inhibits tumor growth remains largely unclear and warrants extensive studies. In this study, we investigated the anticancer effect of DHA on human OS cells. We found that DHA prevents cell proliferation in OS cells effectively. Of note, however, the inhibitory effect of DHA on OS cells is possibly mediated by suppressing the Wnt $/ \beta$-catenin signaling through activation of GSK $3 \beta$ and subsequent reduction of $\beta$-catenin protein.

We found that DHA can reduce metastatic phenotype, as well as expression of MMP9, VEGF and COX2 of OS cells in vitro. MMP9, member of the MMP family, is capable of degrading extracellular matrix and is involved in the metastasis of cancer $(35,36)$. VEGF is a key angiogenic factor that can stimulate vasculogenesis and angiogenesis (37-39). Cancer that expresses VEGF is prone to invade and metastasize $(38,39)$, 
and downregulation of VEGF can attenuate the invasiveness phenotype of malignant tumor $(40,41)$. COX2 is known to be closely associated with tumor growth and metastasis in several types of human tumors $(42,43,25)$. COX2 is unexpressed under normal conditions in most cells, but is upregulated in several types of cancer. The overexpression of COX2 is associated with increased angiogenesis and aggressive invasion in cancer $(43,25)$. In human OS, COX2 is directly associated with OS cell migration and invasion $(25,44)$. Therefore, inhibition of MMP9, VEGF and COX2 expression by DHA may be an important mechanism responsible for the decreased metastatic phenotype in OS cells.

Induction of apoptosis is a common mechanism underlying the anticancer effects of synthetic and natural drugs. Hoechst 33258 staining assay shows that DHA can induce apoptosis in OS cells. Cleaved caspase 3, the activated form of caspase 3 , is regarded as a critical effector in the sequential activation of caspases, and plays a central role in the execution-phase of mitochondrial apoptosis pathway. Our results indicated that DHA can increase the level of cleaved caspase 3 in OS cells, suggesting that the mitochondrial apoptosis pathway is likely involved in DHA-induced apoptosis. $\mathrm{Bad}$ and $\mathrm{Bcl} 2$ belong to the $\mathrm{Bcl} 2$ family members that are critical regulators of apoptosis. However, the role of Bad and $\mathrm{Bcl} 2$ in cell apoptosis is different. Bad is a well-established proapoptotic factor, whereas $\mathrm{Bcl} 2$ is an antiapoptotic molecule known for its ability to inhibit the release of cytochrome $c$ from mitochondria (45). DHA can reduce the levels of $\mathrm{Bcl} 2$ in OS cell lines, whereas it increases Bad protein levels. Therefore, disturbance of the balance between proapoptotic protein (Bad) and antiapoptotic protein $(\mathrm{Bcl} 2)$ by $\mathrm{DHA}$ provides relative evidence to demonstrate the DHA-induced apoptosis in OS cells. Induction of apoptosis of human OS cells by DHA may be an important mechanism responsible for the inhibitory effect on OS cell growth.

It has been reported that the abnormal activation of Wnt/ $\beta$-catenin signaling accounts for tumor initiation of human OS (45-49). Our results showed that DHA can reduce the total protein level of $\beta$-catenin. Overexpression of exogenous $\beta$-catenin alone in OS cells cannot further enhance proliferation and invasion of OS cells. In the present study, we hypothesized that the $\beta$-catenin protein in OS cells is already high enough, therefore exogenous $\beta$-catenin is not necessary to further increase malignant phenotype of OS cells. However, overexpression of exogenous $\beta$-catenin can neutralize the function of DHA, and knockdown of $\beta$-catenin can enhance the growth inhibitory effect of DHA. Therefore, we propose that the effect of DHA on OS cells may, in part, result from targeting $\mathrm{Wnt} / \beta$-catenin signaling.

The Wnt/ $\beta$-catenin signaling is a canonical Wnt pathway that causes an accumulation of $\beta$-catenin and its eventual translocation into the nucleus. The $\beta$-catenin in the nucleus interacts with TCF/LEF family to regulate downstream gene expression (50). Wnt/ $\beta$-catenin pathway plays important roles in embryogenesis and tumorigenesis. Without Wnt ligands, free $\beta$-catenin is rapidly degraded through proteosome machinery promoted by a destruction complex assembled with APC, Axin and GSK3 $\beta$. In the presence of Wnt ligands, this destruction complex function becomes disrupted and the $\beta$-catenin protein becomes stable in the cytoplasm and translocates to the nucleus (50). Following translocation to the nucleus, $\beta$-catenin binds to T-cell factors (TCF) and activates the transcription of specific target genes, including $\mathrm{C}$-myc and cyclin D1 (50). GSK3 $\beta$ is a master protein in the $\mathrm{Wnt} / \beta$-catenin pathway by facilitating phosphorylation and promoting degradation of $\beta$-catenin (50). Catalytic kinase activity of GSK3 $\beta$ is controlled through differential phosphorylation of serine/ threonine residues. Phosphorylation at tyrosine 216 in GSK3 $\beta$ enhances the enzymatic activity of GSK3 $\beta$, while phosphorylation of serine 9 significantly decreases catalytic activity (51). We found that phosphorylation of serine 9 residue in GSK3 $\beta$ is decreased by DHA treatment, which lead to an increased catalytic activity of GSK3 $\beta$. This result indicates that the decrease of $\beta$-catenin protein in OS cells may result from the degradation initialized by GSK3 $\beta$. Our results show that DHA can downregulate DVL proteins in OS cells. DVL proteins are upstream controllers of APC/Axin/GSK3 $\beta$ complex, and are positive mediators of the Wnt/GSK3 $\beta$ pathway. It has been shown that DVL proteins are frequently upregulated and are significantly associated with abnormal elevated $\beta$-catenin protein in several types of cancer (52-54). Moreover, targeted inhibition of DVL proteins decreased $\beta$-catenin protein, reduced TCF-dependent transcription and inhibited cell growth in human cancer $(55,56)$. Although further analysis should be conducted, the decreased DVL proteins by DHA are possibly related to the anticancer effects of DHA by disturbing Wnt/ $\beta$-catenin signaling in OS cells as well.

In conclusion, the present study suggests that DHA can be used as an effective chemotherapy agent for human OS. The proliferation and invasion inhibitory effects of DHA on OS cells may result from inactivating $\mathrm{Wnt} / \beta$-catenin signaling through increasing GSK $3 \beta$ activity and thereby promoting the degradation of $\beta$-catenin. Future studies will be directed at elucidating the exact mechanism of DHA on the degradation of the $\beta$-catenin protein, as well as at combining DHA with other traditional chemotherapy drugs to develop a novel therapy strategy for human OS cancer.

\section{Acknowledgements}

This study was supported in part by research grants from the Natural Science Foundation of China (NSFC 81272006, 31071304), and the National Basic Research Program of China (2011CB707906).

\section{References}

1. Tang N, Song WX, Luo J, Haydon RC and He TC: Osteosarcoma development and stem cell differentiation. Clin Orthop Relat Res 466: 2114-2130, 2008.

2. Whelan JS: Osteosarcoma. Eur J Cancer 33: 1618-1619, 1997.

3. Kager L, Zoubek A, Kastner U, et al: Skip metastases in osteosarcoma: experience of the Cooperative Osteosarcoma Study Group. J Clin Oncol 24: 1535-1541, 2006.

4. Kager L, Zoubek A, Pötschger U, et al: Primary metastatic osteosarcoma: presentation and outcome of patients treated on neoadjuvant Cooperative Osteosarcoma Study Group protocols. J Clin Oncol 21: 2011-2018, 2003.

5. Geller DS and Gorlick R: Osteosarcoma: a review of diagnosis, management, and treatment strategies. Clin Adv Hematol Oncol 8: 705-718, 2010.

6. Ferrari S and Palmerini E: Adjuvant and neoadjuvant combination chemotherapy for osteogenic sarcoma. Curr Opin Oncol 19: 341-346, 2007. 
7. Ayerza MA, Farfalli GL, Aponte-Tinao L and Muscolo DL: Does increased rate of limb-sparing surgery affect survival in osteosarcoma? Clin Orthop Relat Res 468: 2854-2859, 2010.

8. Link MP, Goorin AM, Horowitz M, et al: Adjuvant chemotherapy of high-grade osteosarcoma of the extremity. Updated results of the Multi-Institutional Osteosarcoma Study. Clin Orthop Relat Res 270: 8-14, 1991

9. Bruland OS, Høifødt H, Saeter G, Smeland S and Fodstad O: Hematogenous micrometastases in osteosarcoma patients. Clin Cancer Res 11: 4666-4673, 2005.

10. Cragg GM, Grothaus PG and Newman DJ: Impact of natural products on developing new anti-cancer agents. Chem Rev 109: 3012-3043, 2009.

11. Ji HF, Li XJ and Zhang HY: Natural products and drug discovery. Can thousands of years of ancient medical knowledge lead us to new and powerful drug combinations in the fight against cancer and dementia? EMBO Rep 10: 194-200, 2009.

12. Klayman DL: Qinghaosu (artemisinin): an antimalarial drug from China. Science 228: 1049-1055, 1985.

13. O'Neill PM and Posner GH: A medicinal chemistry perspective on artemisinin and related endoperoxides. J Med Chem 47: 2945-2964, 2004.

14. Singh NP and Lai H: Selective toxicity of dihydroartemisinin and holotransferrin toward human breast cancer cells. Life Sci 70: 49-56, 2001

15. Chen H, Sun B, Wang S, et al: Growth inhibitory effects of dihydroartemisinin on pancreatic cancer cells: involvement of cell cycle arrest and inactivation of nuclear factor- $\kappa \mathrm{B}$. J Cancer Res Clin Oncol 136: 897-903, 2010.

16. He Q, Shi J, Shen XL, An J, et al: Dihydroartemisinin upregulates death receptor 5 expression and cooperates with TRAIL to induce apoptosis in human prostate cancer cells. Cancer Biol Ther 9: 819-824, 2010.

17. Kanno S, Kurauchi K, Tomizawa A, Yomogida S and Ishikawa M: Albumin modulates docosahexaenoic acid-induced cytotoxicity in human hepatocellular carcinoma cell lines. Toxicol Lett 200: 154-161, 2011.

18. Efferth T: Molecular pharmacology and pharmacogenomics of artemisinin and its derivatives in cancer cells. Curr Drug Targets 7: 407-421, 2006

19. Ishiyama M, Tominaga $H$, Shiga M, Sasamoto K, Ohkura $Y$ and Ueno K: A combined assay of cell viability and in vitro cytotoxicity with a highly water-soluble tetrazolium salt, neutral red and crystal violet. Biol Pharm Bull 19: 1518-1520, 1996.

20. Su Y, Luo X, He BC, et al: Establishment and characterization of a new highly metastatic human osteosarcoma cell line. Clin Exp Metastasis 26: 599-610, 2009.

21. Wu K, Yang Q, Mu Y, et al: Berberine inhibits the proliferation of colon cancer cells by inactivating $\mathrm{Wnt} / \beta$-catenin signaling. Int J Oncol 41: 292-298, 2012.

22. Haydon RC, Zhou L, Feng T, et al: Nuclear receptor agonists as potential differentiation therapy agents for human osteosarcoma. Clin Cancer Res 8: 1288-1294, 2002.

23. Park HR and Park YK: Expression of p53 protein, PCNA, and Ki-67 in osteosarcomas of bone. J Korean Med Sci 10: 360-367, 1995.

24. Avnet S, Longhi A, Salerno M, et al: Increased osteoclast activity is associated with aggressiveness of osteosarcoma. Int J Oncol 33: 1231-1238, 2008.

25. Lee EJ, Choi EM, Kim SR, et al: Cyclooxygenase-2 promotes cell proliferation, migration and invasion in U2OS human osteosarcoma cells. Exp Mol Med 39: 469-476, 2007.

26. Kaya M, Wada T, Nagoya S, Sasaki M, Matsumura T and Yamashita T: The level of vascular endothelial growth factor as a predictor of a poor prognosis in osteosarcoma. J Bone Joint Surg Br 91: 784-788, 2009

27. Lu Y and Yagi T: Apoptosis of human tumor cells by chemotherapeutic anthracyclines is enhanced by Bax overexpression. J Radiat Res 40: 263-272, 1999.

28. Seki K, Yoshikawa H, Shiiki K, Hamada Y, Akamatsu N and Tasaka K: Cisplatin (CDDP) specifically induces apoptosis via sequential activation of caspase- $8,-3$ and -6 in osteosarcoma. Cancer Chemother Pharmacol 45: 199-206, 2000.

29. Lamoureux F, Richard P, Wittrant Y, et al: Therapeutic relevance of osteoprotegerin gene therapy in osteosarcoma: blockade of the vicious cycle between tumor cell proliferation and bone resorption. Cancer Res 67: 7308-7318, 2007.

30. Noori S, Hassan ZM, Taghikhani M, Rezaei B and Habibi Z: Dihydroartemisinin can inhibit calmodulin, calmodulindependent phosphodiesterase activity and stimulate cellular immune responses. Int Immunopharmacol 10: 213-217, 2010.
31. Zhao YG, Wang Y, Guo Z, et al: Dihydroartemisinin ameliorates inflammatory disease by its reciprocal effects on Th and regulatory $\mathrm{T}$ cell function via modulating the mammalian target of rapamycin pathway. J Immunol 189: 4417-4425, 2012.

32. Finaurini S, Basilico N, Corbett Y, et al: Dihydroartemisinin inhibits the human erythroid cell differentiation by altering the cell cycle. Toxicology 300: 57-66, 2012.

33. Ba Q, Zhou N, Duan J, et al: Dihydroartemisinin exerts its anticancer activity through depleting cellular iron via transferrin receptor-1. PLoS One 7: e42703, 2012.

34. Firestone GL and Sundar SN: Anticancer activities of artemisinin and its bioactive derivatives. Expert Rev Mol Med 11: e32, 2009.

35. van Kempen LC and Coussens LM: MMP9 potentiates pulmonary metastasis formation. Cancer Cell 2: 251-252, 2002.

36. Groblewska M, Siewko M, Mroczko B and Szmitkowski M: The role of matrix metalloproteinases (MMPs) and their inhibitors (TIMPs) in the development of esophageal cancer. Folia Histochem Cytobiol 50: 12-19, 2012.

37. Claesson-Welsh L and Welsh M: VEGFA and tumour angiogenesis. J Intern Med 273: 114-127, 2013.

38. Lin M, Lin HZ, Ma SP, Ji P, Xie D and Yu JX: Vascular endothelial growth factor-A and $-\mathrm{C}$ : expression and correlations with lymphatic metastasis and prognosis in colorectal cancer. Med Oncol 28: 151-158, 2011

39. Nathanson SD: Insights into the mechanisms of lymph node metastasis. Cancer 98: 413-423, 2003.

40. Carmeliet P: VEGF as a key mediator of angiogenesis in cancer. Oncology 69: 4-10, 2005.

41. Robles Irizarry L, Hambardzumyan D, Nakano I, Gladson CL and Ahluwalia MS: Therapeutic targeting of VEGF in the treatment of glioblastoma. Expert Opin Ther Targets 16: 973-984, 2012.

42. Mao XY, Wang XG, Lv XJ, Xu L and Han CB: COX-2 expression in gastric cancer and its relationship with angiogenesis using tissue microarray. World J Gastroenterol 13: 3466-3471, 2007.

43. Soumaoro LT, Uetake H, Takagi Y, et al: Coexpression of VEGF-C and Cox-2 in human colorectal cancer and its association with lymph node metastasis. Dis Colon Rectum 49: 392-398, 2006

44. Rodriguez NI, Hoots WK, Koshkina NV, et al: COX-2 expression correlates with survival in patients with osteosarcoma lung metastases. J Pediatr Hematol Oncol 30: 507-512, 2008.

45. Reya $\mathrm{T}$ and Clevers $\mathrm{H}$ : Wnt signalling in stem cells and cancer. Nature 434: 843-850, 2005

46. Clevers H: Wnt $/ \beta$-catenin signaling in development and disease. Cell 127: 469-480, 2006.

47. Luu HH, Zhang R, Haydon RC, et al: Wnt/ $/$-catenin signaling pathway as a novel cancer drug target. Curr Cancer Drug Targets 4: 653-671, 2004

48. Haydon RC, Deyrup A, Ishikawa A, et al: Cytoplasmic and/or nuclear accumulation of the $\beta$-catenin protein is a frequent event in human osteosarcoma. Int J Cancer 102: 338-342, 2002.

49. Iwaya K, Ogawa H, Kuroda M, Izumi M, Ishida T, Mukai K: Cytoplasmic and/or nuclear staining of beta-catenin is associated with lung metastasis. Clin Exp Metastasis 20: 525-529, 2003.

50. Luo J, Chen J, Deng ZL, et al: Wnt signaling and human diseases: what are the therapeutic implications? Lab Invest 87: 97-103, 2007.

51. Jope RS, Yuskaitis CJ and Beurel E: Glycogen synthase kinase-3 (GSK3): inflammation, diseases, and therapeutics. Neurochem Res 32: 577-595, 2007

52. Zhao Y, Yang ZQ, Wang Y, et al: Dishevelled-1 and dishevelled-3 affect cell invasion mainly through canonical and noncanonical Wnt pathway, respectively, and associate with poor prognosis in nonsmall cell lung cancer. Mol Carcinog 49: 760-770, 2010.

53. Wei Q, Zhao Y, Yang ZQ, et al: Dishevelled family proteins are expressed in non-small cell lung cancer and function differentially on tumor progression. Lung Cancer 62: 181-192, 2008.

54. Kwan HT, Chan DW, Cai PC, et al: AMPK activators suppress cervical cancer cell grow th through inhibition of DVL3 mediated Wnt/ $\beta$-catenin signaling activity. PLoS One 8: e53597, 2013.

55. Uematsu K, Seki N, Seto T, et al: Targeting the Wnt signaling pathway with dishevelled and cisplatin synergistically suppresses mesothelioma cell growth. Anticancer Res 27: 4239-4242, 2007.

56. Uematsu K, He B, You L, Xu Z, McCormick F and Jablons DM: Activation of the Wnt pathway in non small cell lung cancer: evidence of dishevelled overexpression. Oncogene 22: 7218-7221, 2003. 